\title{
TRIAMETER OF GRAPHS
}

\author{
Angsuman Das \\ Department of Mathematics \\ Presidency University, Kolkata, India \\ e-mail: angsuman.maths@presiuniv.ac.in
}

\begin{abstract}
In this paper, we study a new distance parameter triameter of a connected graph $G$, which is defined as $\max \{d(u, v)+d(v, w)+d(u, w): u, v, w \in$ $V\}$ and is denoted by $\operatorname{tr}(G)$. We find various upper and lower bounds on $\operatorname{tr}(G)$ in terms of order, girth, domination parameters etc., and characterize the graphs attaining those bounds. In the process, we provide some lower bounds of (connected, total) domination numbers of a connected graph in terms of its triameter. The lower bound on total domination number was proved earlier by Henning and Yeo. We provide a shorter proof of that. Moreover, we prove Nordhaus-Gaddum type bounds on $\operatorname{tr}(G)$ and find $\operatorname{tr}(G)$ for some specific family of graphs.
\end{abstract}

Keywords: distance, radio $k$-coloring, Nordhaus-Gaddum bounds.

2010 Mathematics Subject Classification: 05C12.

\section{REFERENCES}

[1] G. Chartrand, D. Erwin and P. Zhang, A graph labeling problem suggested by FM channel restrictions, Bull. Inst. Combin. Appl. 43 (2005) 43-57. 
[2] D. Cvetković and M. Petrić, A Table of connected graphs on six vertices, Discrete Math. 50 (1984) 37-49.

doi:10.1016/0012-365X(84)90033-5

[3] P. Duchet and H. Meyniel, On Hadwiger's number and stability number, Ann. Discrete Math. 13 (1982) 71-74. doi:10.1016/S0304-0208(08)73549-7

[4] O. Favaron and D. Kratsch, Ratios of domination parameters, in: Advances in Graph Theory, V.R. Kulli, (Ed(s)), (Vishwa, Gulbarga, 1991) 173-182.

[5] T.W. Haynes, S.T. Hedetniemi and P.J. Slater, Fundamentals of Domination in Graphs (Marcel Dekker Inc., New York, 1998).

[6] S.T. Hedetniemi and R. Laskar, Connected domination in graphs, in: Graph Theory and Combinatorics, B. Bollobás (Ed(s)), (Academic Press, London 1984), 209-218.

[7] M.A. Henning and A. Yeo, A new lower bound for the total domination number in graphs proving a Graffiti.pc Conjecture, Discrete Appl. Math. 173 (2014) 45-52. doi:10.1016/j.dam.2014.03.013

[8] S.R. Kola and P. Panigrahi, A Lower bound for radio $k$-chromatic number of an arbitrary graph, Contrib. Discrete Math. 10 (2015) 45-56.

[9] M. Laurent, Graphic vertices of the metric polytope, Discrete Math. 151 (1996) 131-151. doi:10.1016/0012-365X(94)00091-V

[10] L. Saha and P. Panigrahi, Antipodal number of some powers of cycles, Discrete Math. 312 (2012) 1550-1557. doi:10.1016/j.disc.2011.10.032

[11] L. Saha and P. Panigrahi, A lower bound for radio $k$-chromatic number, Discrete Appl. Math.192 (2015) 87-100. doi:10.1016/j.dam.2014.05.004

[12] U. Sarkar and A. Adhikari, On characterizing radio $k$-coloring problem by path covering problem, Discrete Math. 338 (2015) 615-620. doi:10.1016/j.disc.2014.11.014

[13] U. Sarkar and A. Adhikari, On relationship between Hamiltonian path and holes in $L(3,2,1)$-coloring of minimum span, Discrete Appl. Math. 222 (2017) 227-234. doi:10.1016/j.dam.2017.01.017

[14] D.B. West, Introduction to Graph Theory (Prentice Hall, Upper Saddle River, 2001).

[15] List of small graphs. http://www.graphclasses.org/smallgraphs.html\#nodes5 http://users.cecs.anu.edu.au/ bdm/data/graphs.html

[16] SageMath. http://www.sagemath.org/

Received 5 June 2018 Revised 25 February 2019 Accepted 25 February 2019 